\title{
Dual Topical Antibiotic Application Prior to Sternotomy Closure Reduces Sternal Wound Infection Rates: A Simple Solution to a Grave Morbidity
}

\author{
Ihab Ali, MD, FRCS (C-Th), Faisal Mourad, MD, FRCS (C-Th) \\ Cardiothoracic Surgery Department, Faculty of Medicine, Ain Shams University, Cairo, Egypt
}

\section{ABSTRACT}

Background: A significant cohort of patients who undergo cardiac surgery suffer from diabetes and atherosclerosis. These patients have impaired tissue perfusion, hence a reduction in antibiotic concentration in the subcutaneous tissues at the side of the mammary artery harvesting. Topical application of gentamicin and vancomycin before wound closure broadens the antibiotic spectrum and reduces the incidence of deep sternal wound infection. In this article, we compare the use of single versus dual application of vancomycin and/or gentamicin in sternotomy wounds in a single tertiary center.

Methods: An observational cohort analysis with three sequential patient groups $(\mathrm{N}=2550)$ was performed at Ain Shams University Hospital in Cairo. A control group $(\mathrm{N}=850)$, vancomycin only group $(\mathrm{N}=850)$, and vancomycin plus gentamicin group $(\mathrm{N}=850)$ were included in the study, during the three-year period from January 2017 to December 2019. Patients who had minimal access surgery were excluded from this study. The presence of an infected postoperative sternotomy wound was assessed in all patients.

Results: The presence of an infected sternotomy wound (El Oakley class 2B) was present in 38 patients $(4.5 \%)$ in the control group, in 19 patients $(2.2 \%)$ in the vancomycin group, and in nine patients $(1.1 \%)$ in the dual antibiotic group, respectively $(P<.001)$. In contrast to the usual, we had a proliferous growth of gram-negative organisms 29 $(3.4 \%)$ in the control group, $10(1.2 \%)$ in the vancomycin group, and five $(0.6 \%)$ in the dual antibiotic group, respectively $(P<.001)$.

Conclusion: Deep sternal wound infection is a major cause of post-cardiac surgery morbidity and prolonged hospital stay. Adding the simple step of topical application of vancomycin and gentamicin to the sternotomy wound at the end of the procedure appeared to significantly reduce deep wound infection rates.

Received April 10, 2021; received in revised form May 5, 2021; accepted May 5, 2021.

Correspondence: Prof. Ihab Ali, MD, FRCS (C-Th), Associate Professor of Cardio-thoracic Surgery, Cardiothoracic Surgery Department, Faculty of Medicine, Ain Shams University Hospital, Abbasyia Square, Cairo, Egypt, P.O. Code 11355; 00201279410660 (e-mail: dribababdelrazek@ botmail.com).

\section{INTRODUCTION}

Sternotomy infections are still a major complication in cardiac surgery affecting patient morbidity and mortality [Borger 1998; Fleck 2006; Velebit 2010]. A significant cohort of patients who undergo cardiac surgery suffer from diabetes and atherosclerosis. Those patients have impaired tissue perfusion, hence a reduction in antibiotic concentration in the subcutaneous tissues at the side of the mammary artery harvesting. This leads to an insufficient antibiotic coverage of common pathogens, despite giving appropriate doses of perioperative antibiotic prophylaxis [Andreas 2015; Andreas 2013].

Local antibiotic application to sternal edges could be the solution. It results in antibiotic concentrations in the wound that are much higher than those achieved when antibiotics systemically are administered [Halasz 1977]. The idea of applying vancomycin directly into the wound first was introduced in 1989 in order to prevent deep sternal wound infections [Vander Salm 1989].

According to the guidelines released by the American Association for Thoracic Surgery (AATS) in 2016, topical application of antibiotics to sternal edges after opening and before closing the sternum is mandatory in all sternotomy procedures (Class I Recommendation; Level of Evidence = B) [Lazar 2016]. According to the Society of Thoracic Surgeons Adult Cardiac Surgery Database, implementation of these guidelines has led to a reduction in the incidence of DSWI down to $0.3 \%$ in coronary artery bypass operations [D'Agostino 2018].

The combination of gentamicin and vancomycin broadens the antibiotic spectrum and its application using a sponge reduces the incidence of deep sternal wound infection [Kowalewski 2015; Friberg 2007].

In this article, we compare the use of single versus dual application of vancomycin and/or gentamicin in sternotomy wounds in a single tertiary center.

\section{MATERIALS AND METHODS}

\section{Ethical considerations}

The study was reviewed and approved by the Institutional Review Board of Faculty of Medicine, Ain Shams University (Ain Shams University Protocol Record: FMASU R 72/2020) and was conducted in accordance with the Declaration of Helsinki. 
Table 1. Comparison between the three study groups regarding patient demographics

\begin{tabular}{|c|c|c|c|c|c|}
\hline & Control $(\mathrm{N}=850)$ & Vanc only $(\mathrm{N}=850)$ & Vanc + Gent $(N=850)$ & Tests $-\mathrm{f} / \mathrm{X}^{2}$ & Tests - P-value \\
\hline Age & $63.69 \pm 5.40$ & $64.19 \pm 5.31$ & $63.60 \pm 5.85$ & 2.787 & .062 \\
\hline Female & $280(32.9 \%)$ & $263(30.9 \%)$ & $278(32.7 \%)$ & 0.931 & .628 \\
\hline Male & $570(67.1 \%)$ & $587(69.1 \%)$ & $572(67.3 \%)$ & & \\
\hline Weight & $84.30 \pm 10.84$ & $84.38 \pm 10.87$ & $83.77 \pm 10.63$ & 0.787 & .455 \\
\hline Body mass index & $27.95 \pm 4.06$ & $28.11 \pm 4.11$ & $27.86 \pm 3.95$ & 0.859 & .424 \\
\hline Additive Euro SCORE & $3.91 \pm 7.74$ & $3.78 \pm 7.50$ & $3.67 \pm 7.44$ & 1.497 & .224 \\
\hline Logistic Euro SCORE & $6.10 \pm 12.65$ & $6.09 \pm 12.48$ & $5.93 \pm 12.24$ & 0.966 & .381 \\
\hline Euro SCORE II & $3.65 \pm 7.17$ & $3.96 \pm 7.41$ & $3.67 \pm 7.13$ & 1.408 & .245 \\
\hline Coronary artery disease & $528(62.9 \%)$ & $533(62.7 \%)$ & $530(62.4 \%)$ & 0.064 & .969 \\
\hline Cerebrovascular disease & $177(20.8 \%)$ & $173(20.4 \%)$ & $175(20.6 \%)$ & 0.058 & .972 \\
\hline Peripheral vascular disease & $126(14.8 \%)$ & $134(15.8 \%)$ & $129(15.2 \%)$ & 0.297 & .862 \\
\hline COPD & $148(17.4 \%)$ & $153(18.0 \%)$ & $151(17.8 \%)$ & 0.102 & .950 \\
\hline On corticosteroids & $82(9.6 \%)$ & $77(9.1 \%)$ & $80(9.4 \%)$ & 0.175 & .916 \\
\hline Creatinine $\mathrm{mg} / \mathrm{dl}$ & $1.04 \pm 0.14$ & $1.04 \pm 0.12$ & $1.05 \pm 0.13$ & 1.208 & .299 \\
\hline Previous valve surgery & $61(7.2 \%)$ & $68(8.0 \%)$ & $65(7.6 \%)$ & 0.413 & .813 \\
\hline Previous bypass surgery & $53(6.2 \%)$ & $41(4.8 \%)$ & $50(5.9 \%)$ & 1.722 & .423 \\
\hline
\end{tabular}

$P$-values $<.05$ were considered statistically significant. COPD, chronic obstructive pulmonary disease

\section{Study design}

An observational cohort analysis with three sequential patient groups was performed at Ain Shams University Hospital in Cairo.

\section{Study population}

All consecutive patients undergoing cardiac surgery via full sternotomy during a three-year period $(\mathrm{N}=2550)$. Patients in the control group $(\mathrm{N}=850)$ were operated on between January 2017 and December 2017. Patients with the antibiotic closure protocol (vancomycin only group) $(\mathrm{N}=850)$ were operated on between January 2018 and December 2018, while patients with the antibiotic closure protocol (vancomycin plus gentamicin group) $(\mathrm{N}=850$ ) were operated on between January 2019 and December 2019. Patients who had minimal access surgery were excluded from this study.

\section{Chest closure procedure, new method:}

The introduction of the new closure procedure was adopted after educating and training all members of staff and was applied in every patient without known allergies to vancomycin or gentamicin. Our hypothesis was based on the recommendations made by EurAsia Heart for the reduction of sternal wound infections [Vogt 2016].
Vancomycin has the advantage of being a cheap drug with no side effects, when applied topically. It provides cover against gram-positive bacteria and clostridia organisms [Lazar 2018]. Vancomycin powder (3 g) was sprinkled onto the subcutaneous tissue and applied directly onto the sternal bone marrow, using a swab. It was put into the sternum halves similar to bone wax directly prior to sternal wiring. Bone wax, if applied, was removed prior to vancomycin application. Gentamicin $(160 \mathrm{mg})$ was mixed with $500 \mathrm{ml}$ of saline and irrigated on to the wound directly prior to subcutaneous closure. This step was added to the vancomycin application, due to the rare clinical application of gentamicin, leading to a low rate of antibiotic resistance, and due to the high antibacterial activity after topical application [Junker 2015]. Gentamicin also was added as per our infection control department recommendation. Seven or eight simple wire applications were recommended for every patient, when appropriate.

Other measures of infection prophylaxis were not changed by this protocol. Therefore, all three groups similarly were treated with the following prevention steps:

Every patient underwent nasal decontamination (Prontoderm VR, B. Braun Medical Inc., Melsungen, Germany) on admission. Every patient received intravenous 
Table 2. Comparison between the three study groups regarding operative data

\begin{tabular}{|c|c|c|c|c|c|}
\hline \multicolumn{6}{|l|}{ Bypass and/or valve } \\
\hline Valve surgery & $290(34.1 \%)$ & $306(36.0 \%)$ & $288(33.9 \%)$ & 4.328 & .363 \\
\hline Bypass and valve & $213(25.1 \%)$ & $204(24.0 \%)$ & $238(28.0 \%)$ & & \\
\hline LIMA & $410(73.2 \%)$ & $391(71.9 \%)$ & $409(72.8 \%)$ & & \\
\hline RIMA & $33(5.9 \%)$ & $38(7 \%)$ & $28(5 \%)$ & 5.801 & .446 \\
\hline BIMA & $28(5 \%)$ & $33(6 \%)$ & $23(4.1 \%)$ & & \\
\hline No IMA & $89(15.9 \%)$ & $82(15.1 \%)$ & $102(18.1 \%)$ & & \\
\hline Redo surgery & $136(16.0 \%)$ & $144(16.9 \%)$ & $127(14.9 \%)$ & 1.269 & .530 \\
\hline LOS (days) & $2.62 \pm 9.11$ & $2.82 \pm 9.06$ & $2.67 \pm 9.31$ & 2.050 & .129 \\
\hline Thirty-day mortality & $26(3.1 \%)$ & $34(4.0 \%)$ & $34(4.0 \%)$ & 1.414 & .493 \\
\hline
\end{tabular}

$P$-values $<.05$ were considered statistically significant. LIMA, left internal mammary artery;

RIMA, right internal mammary artery; BIMA, bilateral internal mammary artery; BMI: body mass index; CPB, cardiopulmonary bypass; XCT, cross-clamp time; ICU, intensive care unit; LOS, length of hospital stay

antibiotic prophylaxis during surgery. Our intravenous antibiotic strategy consists of $2 \mathrm{~g}$ cefazolin applied one hour prior to skin incision and an additional dose of $2 \mathrm{~g}$ cefazolin was given during skin closure [Andreas 2013]. A sternal spreader with wide blades routinely was used to avoid sternal fracture or perfusion impairment during surgery.

Statistical data: Patient data were recorded and recalled using our red cap data management system. We waived patient consent after obtaining ethical committee approval as we changed the standard of care, according to the new protocol. All wound infection cases were recorded and followed up using our infection control department and were classified using El Oakley classification [El Oakley 1996]. We included only patients with deep sternal wound infections El Oakley class $2 \mathrm{~B}$ and higher.

Statistical analysis: Statistical presentation and analysis of the present study was conducted, using the mean, standard deviation, according to the computer program SPSS for Windows. ANOVA test was used for comparison among different times in the same group in quantitative data, and qualitative data was expressed as frequency (number and percentage) by chi-square test by (IBM SPSS Statistics for Windows, Version 20.0, IBM Corp., Armonk, NY). A $P$-value $<.05$ was considered significant.

Sample size justification: Sample size was calculated using PASS program, setting the type- 1 error $(\alpha)$ at 0.05 and the power $(1-\beta)$ at 0.8 . The current study is planned to test whether the infection rate in each of the two study treatment groups (vancomycin, and combined vancomycin and gentamicin) are significantly different from the control-group (no treatment). Results from previous study [Andreas 2017] showed that the infection rate among the combined treatment group was $1.2 \%$, while it is assumed to be $2 \%$ in the vancomycin treatment group and $4.7 \%$ in the control group. Based on this, the needed sample size is 850 cases per each group. Hence, the total sample size is 2550 .

\section{RESULTS}

The annual workload in our hospital is around 1200 cases per year. Based on our sample size calculations, we divided the patients into three equal groups with 850 patients (33.3\%) operated upon in each category during the study period. All patients in the three groups demographically were matched (Table 1).

All patients were operated upon using full sternotomy. Minimally invasive procedures were excluded from our study population. We included all comers whether emergency or redo cases, hence the higher Euro score II average 7.1 +/- 3.6. Operative variability CABG, valve, $\mathrm{CABG}$ and valve surgery was matched in the three groups, $P=.36$. With regard to the procedural complexity as reflected on the $\mathrm{CPB}$ time and cross-clamp time, all three groups equally were matched $P=$ .45 and $P=.35$, respectively. There was no significant statistical difference in the 30-day mortality rate ranging between $3 \%-4 \%$, respectively $(P=.49)$ (Table 2$)$.

There was a significant difference in the incidence of deep sternal wound infection (El Oakley 2B). This was evident in 
Table 3. Comparison between the three study groups regarding deep sternal wound infection rates

\begin{tabular}{|c|c|c|c|c|c|}
\hline Total infection rate & 38 (4.5\%) & $19(2.2 \%)$ & $9(1.1 \%)$ & 20.251 & $<.001 * *$ \\
\hline
\end{tabular}

Table 4. Comparison between the three study groups regarding the causative organisms

\begin{tabular}{lccccc}
\hline & Control $(\mathrm{N}=850)$ & Vanc only $(\mathrm{N}=850)$ & Vanc + Gent $(\mathrm{N}=850)$ & Chi-square - $\mathrm{X}^{2}$ & Chi-square $-P$-value \\
\hline Gram-negative & $29(3.4 \%)$ & $10(1.2 \%)$ & $5(0.6 \%)$ & 22.745 & $<.001 *$ \\
Gram-positive & $6(0.7 \%)$ & $5(0.6 \%)$ & $3(0.4 \%)$ & 1.012 & .603 \\
Fungus & $3(0.4 \%)$ & $4(0.5 \%)$ & $1(0.1 \%)$ & 1.755 & .416
\end{tabular}

38 patients $(4.5 \%)$ in the control group, in 19 patients $(2.2 \%)$ in the vancomycin group, and nine patients $(1.1 \%)$ in the dual antibiotic group, respectively $(P<.001)$ (Table 3$)$.

In contrast to the usual, we had a proliferous growth of gram-negative organisms $29(3.4 \%)$ in the control group, 10 $(1.2 \%)$ in the vancomycin group, and five $(0.6 \%)$ in the dual antibiotic group, respectively $(P<.001)$ (Table 4$)$.

All patients with deep sternal wound infection (El Oakley 2B) were placed on negative pressure wound therapy (NPWT) of variable duration, ranging between 48 hours and 256 days mean 31.4 days $\mathrm{SD}+/-3.3$ before a definitive procedure was made. Sternal rewiring was the most common surgical procedure performed to restore sternal integrity $12(1.4 \%), 10$ $(1.2 \%)$, and $4(0.4 \%)$, respectively (Table 5$)$.

\section{DISCUSSION}

El Oakley and Wright classified complications related to sternotomy wound healing in their review as follows: 1-Mediastinal dehiscence: sternal malunion with no clinical or microbiological signs of infection. 2-Mediastinal wound infection: clinically or microbiologically infected presternal tissue and sternal osteomyelitis regardless mediastinal sepsis and regardless sternal stability. This type is further classified into type A: superficial infection: limited to subcutaneous tissue, and type B: deep infection: osteomylitic sternum [El Oakley 1996].

In this study, we monitored the incidence of deep sternal wound infection (El Oakley type $2 \mathrm{~B}$ ), which is a major cause of post-cardiac surgery morbidity and prolonged hospital stay. Negative pressure wound therapy is considered effective but time consuming, and it may not always provide a definitive cure for those patients [Tarzia 2014]. The golden rule in this scenario is prevention better than cure.

Prior to the introduction of topical antibiotics to our sternal closure technique, our deep sternal wound infection rate was $4.5 \%$, which was in line with other reports [Jonkers 2003; Sharma 2004; Antunes 1997; Schimmer 2016]. However, by adding the simple step of topical application of vancomycin and gentamicin to the sternotomy wound at the end of the procedure, the deep wound infection rate drastically dropped to $1.1 \%(P<.001)$.
Our findings were consistent with the findings of several studies: The study by Vander Salm et al concluded that topical vancomycin caused a drop in the incidence of sternotomy wound infections from $3.6 \%$ to $0.5 \% ; P=.02$ [Vander Salm 1989]. In his recent meta-analysis published in 2017, Kowalewski et al proved that the use of vancomycin paste resulted in a decrease in the incidence of deep sternal wound infections by $76 \%$ [Kowalewski 2017].

Topical vancomycin paste decreased the incidence of sternal wound infections to $0.5 \%$, in a study done in retrospect by Arruda et al and involved more than 1000 patients [Arruda 2008].

In a study that included 3000 patients, Lazar et al demonstrated that vancomycin applied as a slurry to sternal edges, together with systemic antibiotics and strict blood sugar control, resulted in disappearance of superficial ( 0 versus $1.6 \%$; $P<.001)$ and deep ( 0 versus $0.7 \% ; P=.005)$ wound infections [Lazar 2014].

The topical effectiveness of vancomycin and gentamicin previously was demonstrated in other reports [Friberg 2007; Junker 2015]. The increased incidence of gram-negative organisms in our studied sample $(P<.001)$ could be widely attributed to the isolation of pseudomonas aeruginosa from the hospital's air conditioning filters. The significant reduction in deep wound infection upon adding gentamicin wash before sternal closure may be explained by the effectiveness of gentamicin against gram-negative organisms.

Our findings in this prospect are consistent with the results of the meta-analysis published in 2015, where Kowalewski et al demonstrated that gentamicin-collagen sponges resulted in a $40 \%$ drop in the incidence of superficial and deep sternotomy wound infections [Kowalewski 2015].

None of the patients in our study had adverse effects from the application of the topical antibiotics. This also is consistent with Lazar et al who concluded that vancomycin, when applied locally, did not cause any postoperative drug resistant infections or kidney injury [Lazar 2011].

Andreas et al demonstrated the reduction of infection rate using vancomycin and gentamicin paste, but we were able to show similar results just by using direct application of vancomycin powder and gentamicin irrigation wash [Andreas 2017]. 
Table 5. Comparison between the three study groups regarding wound interventions

\begin{tabular}{lccc}
\hline & $\begin{array}{c}\text { Control } \\
(\mathrm{N}=850)\end{array}$ & $\begin{array}{c}\text { Vanc only } \\
(\mathrm{N}=850)\end{array}$ & $\begin{array}{c}\text { Vanc + Gent } \\
(\mathrm{N}=850)\end{array}$ \\
\hline Rewiring & $12(1.4 \%)$ & $10(1.2 \%)$ & $4(0.4 \%)$ \\
Pectoral muscle flap & $6(0.7 \%)$ & $3(0.4 \%)$ & $1(0.2 \%)$ \\
Rectus muscle flap & $4(0.4 \%)$ & $1(0.2 \%)$ & $0(0 \%)$ \\
Subcutaneous closure & $9(1.0 \%)$ & $1(0.2 \%)$ & $2(0.2 \%)$ \\
No surgical closure & $7(0.8 \%)$ & $4(0.4 \%)$ & $2(0.2 \%)$
\end{tabular}

Some researchers studied using alternative antibiotics locally. In his study done retrospectively, De Santo et al concluded that patients receiving local rifampicin wash of the mediastinum and sternal edges showed significant reduction in the incidence of deep sternal wound infection $(0.3 \%$ versus $2.1 \%, P=.0391)$. ICU stay, hospital stay, and operative mortality were similar between the groups [De Santo 2020].

Diagnosing DSWI is a challenging task combining many modalities in order to confirm the diagnosis. The Centers for Disease Control and Prevention (CDC) have set criteria for confirming the diagnosis: 1) Isolation of microorganisms from wound cultures intended for clinical diagnosis or treatment; 2) Evidence of mediastinitis on gross or microscopic histopathological examination; 3) Clinical signs and symptoms in the form of fever $\left(>38.0^{\circ} \mathrm{C}\right)$, chest pain, or sternal instability, and at least one of the following: purulent drainage from the mediastinal area or mediastinal widening on imaging test. Other signs of DSWI include cellulitis, wound dehiscence, which could be accompanied by a systemic inflammatory response in the form of tachycardia and hypotension [Horan 2008]. These manifestations usually present within 30 days of cardiac surgery [Robicsek 2000].

Many surgical options exist in current practice, however there is a lack of consensus on optimal surgical management [Schimmer 2016]. In the 1960s, traditional surgical modalities were established in the form of wound debridement, primary sternal closure, and mediastinal catheter irrigation with antibiotic or antiseptic solution [Abu-Omar 2017; Cotogni 2015]. Despite its wide adaptation for many years, this technique failed to achieve satisfactory results thus alternative measures were adopted, which involved surgical debridement, open dressing and secondary closure, with or without reconstruction with vascularized soft tissue flaps such as greater momentum or pectoral muscles [Singh 2011; Atkins 2011].

By the late 1990s, the emergence of negative pressure wound therapy (NPWT; also known as vacuum-assisted closure [VAC]) helped improve the results of mediastinitis treatment. The continual drainage of bacteria, debris, and exudates by negative wound pressure enhances microcirculation and accelerates tissue granulation [Jones 1997]. The mass filling effect of the foam stabilizes and approximates the sternal wound edge. Prompted by its increasing use, several studies have found the clinical effect of VAC to be comparable with traditional closed drainage or open packing, with improvement in sternal wound healing, reinfection rates, length of ICU stay, and possibly mortality [Morykwas 1997; De Feo 2011].

The application of VAC should be approached with caution as the VAC system itself can be a reservoir for microorganisms and may lead to emergence of multi-resistant microorganisms, such as gram-negative bacilli and yeasts (Candida $s p p$.) In a study by Yusuf et al, they demonstrated that 68 foams were cultured and after sonication procedure they found that in $65(97 \%)$ foams, at least one bacterial species was found. The bacterial load was ranging from 104 to 106 $\mathrm{CFU} / \mathrm{ml}$ [Sjögren 2005].

All our patients with DSWI were placed on VAC therapy, the duration of which was variable ranging between 48 hours and 256 days mean 31.4 days $\mathrm{SD}+/-3.3$. This was in line with what Berdasj and colleagues described, where they found variations in clinical practice, which included early closure within 48 hours to multiple debridement until bacteriology samples were negative. We tried to keep our VAC therapy duration as short as possible followed by secondary closure to reduce the risk of secondary infection from bacterial inoculation and environmental exposure of the open wound [Yusuf 2013].

Study limitation: This is a single-center study.

\section{CONCLUSION}

Deep sternal wound infection is a major cause of postcardiac surgery morbidity and prolonged hospital stay. Adding the simple step of topical application of vancomycin and gentamicin to the sternotomy wound toward the end of the procedure showed to significantly reduce deep sternal wound infection rates.

\section{REFERENCES}

Abu-Omar Y, Kocher GJ, Bosco P, et al. 2017. European association for cardiothoracic surgery expert consensus statement on the prevention and management of mediastinitis. Eur J Cardiothorac Surg. 2017;51(1):10-29.

Andreas M, Muckenhuber M, Hutschala D, Kocher A, Thalhammer F, Vogt P, et al. 2017. Direct sternal administration of Vancomycin and Gentamicin during closure prevents wound infection. Interactive cardiovascular and thoracic surgery, 25(1), 6-11.

Andreas M, Zeitlinger M, Hoeferl M, Jaeger W, Zimpfer D, Hiesmayr $\mathrm{JM}$, et al. 2013. Internal mammary artery harvesting influences antibiotic penetration into presternal tissue. Ann Thorac Surg. 95:1323-9; discussion 29-30.

Andreas M, Zeitlinger M, Wisser W, Jaeger W, Maier-Salamon A, Thalhammer F, et al. 2015. Cefazolin and linezolid penetration into sternal cancellous bone during coronary artery bypass grafting. Eur J Cardiothorac Surg. 48:758-64.

Antunes PE, Bernardo JE, Eugenio L, de Oliveira JF, Antunes MJ. 1997. Mediastinitis after aorto-coronary bypass surgery. Eur J Cardiothorac Surg. 12:443-9.

Arruda MVF, Braile DM, Joaquim MR, Suzuki FA, Alves RH. 2008. The use of vancomycin paste for sternal hemostasis and mediastinitis prophylaxis. Rev Bras Circ Cardiovasc. 23:35-9. 
Atkins BZ, Onaitis MW, Hutcheson KA, Kaye K, Petersen RP, Wolfe WG. 2011. Does method of sternal repair influence long-term outcome of postoperative mediastinitis? Am J Surg. 202(5):565-567.

Borger MA, Rao V, Weisel RD, Ivanov J, Cohen G, Scully HE, et al. 1998. Deep sternal wound infection: risk factors and outcomes. Ann Thorac Surg. 65:1050-6.

Cotogni P. 2015. Deep sternal wound infection after cardiac surgery: evidences and controversies. World J Crit Care Med. 4(4):265-273.

D'Agostino RS, Jacobs JP, Bardhwar V, et al. 2018. The Society of Thoracic Surgeons Adult Cardiac Surgery Database: 2018 update on outcomes and quality. Ann Thorac Surg. 105:15-23.

De Feo M, Vicchio M, Santè P, Cerasuolo F, Nappi G. 2011. Evolution in the treatment of mediastinitis: single-center experience. Asian Cardiovasc Thorac Ann. 19(1):39-43.

De Santo LS, Rubino AS, Torella M, et al. 2020. Topical rifampicin for prevention of deep sternal wound infections in patients undergoing coronary artery bypass grafting. Sci Rep10, 7400.

El Oakley RM, Wright JE. 1996. Postoperative mediastinitis: classification and management. Ann Thorac Surg. 61:1030-6.

Fleck T, Gustafsson R, Harding K, Ingemansson R, Lirtzman MD, Meites HL, et al. 2006. The management of deep sternal wound infections using vacuum assisted closure (V.A.C.) therapy. Int Wound J. 3:273-80.

Friberg O, Svedjeholm R, Kallman J, Soderquist B. 2007. Incidence, microbiological findings, and clinical presentation of sternal wound infections after cardiac surgery with and without local gentamicin prophylaxis. Eur J Clin Microbiol Infect Dis. 26:91-7.

Halasz NA. 1977. Wound infection and topical antibiotics: the surgeon's dilemma. Arch Surg. 112:1240-4.

Horan TC, Andrus M, Dudeck MA. 2008. CDC/NHSN surveillance definition of healthcare-associated infection and criteria for specific types of infections in the acute care setting. Am J Infect Control. 36(5):309-332.

Jones G, Jurkiewicz MJ, Bostwick J, et al. 1997. Management of the infected median sternotomy wound with muscle flaps. The Emory 20-year experience. Ann Surg. 225(6):766-776.

Jonkers D, Elenbaas T, Terporten P, Nieman F, Stobberingh E. 2003. Prevalence of 90 -days postoperative wound infections after cardiac surgery. Eur J Cardiothorac Surg. 23:97-102.

Junker JP, Lee CC, Samaan S, Hackl F, Kiwanuka E, Minasian RA, et al. 2015. Topical delivery of ultrahigh concentrations of gentamicin is highly effective in reducing bacterial levels in infected porcine full-thickness wounds. Plast Reconstr Surg. 135:151-9.

Kowalewski M, Pawliszak W, Zaborowska K, Navarese EP, Szwed KA, Kowalkowska ME, et al. 2015. Gentamicin-collagen sponge reduces the risk of sternal wound infections after heart surgery: meta-analysis. J Thorac Cardiovasc Surg. 149:1631-40.e1-6.

Kowalewski M, Raffa GM, Szwed KA, Anisimowicz L. 2017. Meta-analysis to assess the effectiveness of topically used vancomycin in reducing sternal would infections after cardiac surgery. J Thorac Cardiovasc Surg.
154:1320-3.

Lazar HL. 2018. A review of the AATS guidelines for the prevention and management of sternal wound infections. Indian J Thorac Cardiovasc Surg. Dec;34(Suppl 3):349-354.

Lazar HL, Barlam T, Cabral H. 2011. The effect of topical vancomycin applied to sternotomy incisions on postoperative serum vancomycin levels. J Card Surg. 26:461-5.

Lazar HL, Ketchedjian A, Haime M, Karlson K, Cabral H. 2014. Topical vancomycin in combination with perioperative antibiotics and tight glycemic control helps to eliminate sternal wound infections. J Thorac Cardiovasc Surg. 148:1035-40.

Lazar HL, Salm VT, Engelman R, Orgill D, Gordon S. 2016. Prevention and management of sternal wound infections. J Thorac Cardiovasc Surg. 152:962-72.

Morykwas MJ, Argenta LC, Shelton-Brown EI, McGuirt W. 1997. Vacuum-assisted closure: a new method for wound control and treatment: animal studies and basic foundation. Ann Plast Surg. 38(6):553-562.

Robicsek F. 2000. Postoperative sterno-mediastinitis. Am Surg. 66(2):184-192.

Schimmer C, Gross J, Ramm E, Morfeld BC, Hoffmann G, Panholzer B, et al. 2016. Prevention of surgical site sternal infections in cardiac surgery: a two-centre prospective randomized controlled study. Eur J Cardiothorac Surg. 51:67-72.

Sharma M, Berriel-Cass D, Baran J Jr. 2004. Sternal surgical-site infection following coronary artery bypass graft: prevalence, microbiology, and complications during a 42-month period. Infect Control Hosp Epidemiol. 25:468-71.

Singh K, Anderson E, Harper JG. 2011. Overview and management of sternal wound infection. Semin Plast Surg. 25(1):25-33.

Sjögren J, Gustafsson R, Nilsson J, Malmsjö M, Ingemansson R. 2005 Clinical outcome after poststernotomy mediastinitis: vacuum-assisted closure versus conventional treatment. Ann Thorac Surg. 79(6):2049-2055.

Tarzia V, Carrozzini M, Bortolussi G, Buratto E, Bejko J, Comisso M, Mescola V, Penzo V, Guarino M, De Franceschi M, Pagnin C, Castoro M, Guglielmi C, Testolin L, Bottio T, Gerosa G. 2014. Impact of vacuumassisted closure therapy on outcomes of sternal wound dehiscence, Interactive Cardiovascular and Thoracic Surgery, Volume 19, Issue 1, Pages 70-75.

Vander Salm TJ, Okike ON, Pasque MK, Pezzella AT, Lew R, Traina $\mathrm{V}$, et al. 1989. Reduction of sternal infection by application of topical vancomycin. J Thorac Cardiovasc Surg. 98:618-22.

Velebit V. 2010. Deep sternal wound infection—still a challenge. Eur J Cardiothorac Surg. 37:749.

Vogt P. 2016. EurAsia Heart. http://www.eurasiaheart.ch/de/eurasiaheart/science-research.php (25 September, date last accessed).

Yusuf E, Jordan X, Clauss M, Borens O, Mäder M, Trampuz A. 2013. High bacterial load in negative pressure wound therapy (NPWT) foams used in the treatment of chronic wounds. Wound Repair Regen. 2013;21(5):677-681. 